\title{
SEXO, PESO E CONFORMAÇÃO ANATÔMICA DO OLHO SOBRE CÁLCULO DE PODER DIÓPTRICO DE LENTES INTRA-OCULARES NO CÃO
}

\author{
SEX, ANATOMY AND WEIGHT ON INTRAOCULAR LENS DIOPTRIC POWER \\ CALCULATION IN DOGS
}

\section{Gabriela Rodrigues Sampaio ${ }^{1}$ José Joaquim Titton Ranzani $^{2}$ Silvana Artioli Schellini $^{3}$}

\section{RESUMO}

A catarata, especialmente na espécie canina, é uma doença oftálmica importante. Seu tratamento é eminentemente cirúrgico, mas a perda do cristalino resulta em alta hipermetropia, e a visão fica comprometida. A correção pode ser feita com a utilização de lente intra-ocular (LIO). Este estudo teve por objetivo colher dados que pudessem fornecer subsídios métricos para a produção de LIOs a serem aplicadas em cães facectomizados, visando a restauração de visão adequada nos animais acometidos por catarata. Foram selecionados 120 cães de ambos os sexos, sadios ao exame clínico e sem evidência de doença ocular ao exame oftálmico de rotina. Foram realizados estudos ceratométrico e biométrico, pela ultra-sonografia modoA. Uma fórmula específica foi utilizada para a determinação do poder dióptrico de uma LIO utilizada para substituir o cristalino extraído. O poder dióptrico da LIO foi correlacionado com o peso e o sexo dos animais, e entre os olhos direito e esquerdo. Baseado nestas correlações, concluiu-se que o poder dióptrico adequado para uma LIO a ser implantada em um cão facectomizado é influenciado principalmente pelo peso do animal, apesar de apresentar discretas diferenças quando comparado entre os olhos direito e esquerdo e entre machos $e$ fêmeas.

Palavras-chave: cão, olho, ceratometria, biometria, ultrasonografia modo-A, lente intra-ocular.

\section{SUMMARY}

Cataract is an important ophthalmic disease in dogs. Treatment is often surgical, but the removal of the lens produces hypermetropy and vision is impaired. Correction is usually performed with an intraocular lens (IOL). This study had the purpose of obtaining data for intraocular lens production in order to use in aphakic dogs, because cataracts is a very common ocular disease in this species, and surgical treatment is mandatory. One hundred and twenty dogs of both sexes, clinicaly healthy and free from ophthalmic disease were used. A keratometric study and a biometric study, using A-mode ultrasonography, were used. Some of these measures were determined using a specific formula for evaluation of the IOL dioptric power, to substitute the affected lens. The IOL dioptric power was correlated with weight and sex and left and right eyes. Although some differences were observed between left and right eyes and males and females, body weight was the most important variable to influence IOL dioptric power in dogs.

Key words: dog, eye, keratometry, biometry, A-mode ultrasonography, intraocular lens.

\section{INTRODUÇÃO}

Das doenças oculares dos cães, a catarata ocupa lugar de destaque. Catarata é o nome que se dá à opacidade do cristalino, resultando em baixa visão, chegando a impossibilitar o contato visual com o meio ambiente. Seu único tratamento é cirúrgico, mas a remoção desta lente torna o olho alto hipermétrope. Relatos sobre facectomia em cães iniciaram nos anos 50 e 60 , ficando os cirurgiões satisfeitos com a visão afácica hiperópica dos pacientes, a qual possibilita evitar colisões e reconhecer pessoas e objetos. A correção desta alta

\footnotetext{
${ }^{1}$ Professor Assistente, Departamento de Medicina Veterinária, Universidade Federal de Lavras (UFLA), CP 37, 37200-000, Lavras, MG. E-mail:gabsampa@ufla.br. Autor para correspondência.

${ }^{2}$ Professor Assistente, Doutor, Departamento de Cirurgia e Anestesiologia Veterinária, Faculdade de Medicina Veterinária e Zootecnia, Universidade Estadual Paulista (UNESP).

${ }^{3}$ Professor Adjunto, Departamento de Oftalmologia e Otorrinolaringologia, Faculdade de Medicina, (UNESP).
} 
hipermetropia e obtenção de visão mais acurada são possíveis com o uso de LIOs em substituição ao cristalino extraído (GAIDDON et al., 1991).

A primeira citação de aplicação de LIO foi feita em humanos, por Ridley, apud RUBIN \& HOPE (1996), e data de 1949. Essa descoberta tornou rotineiro o uso de LIOs em humanos, mas, nos animais, a forma do cristalino, por variar nas diversas espécies, torna a oftalmologia veterinária carente de informações mais precisas sobre esse assunto. Não se tem notícia da existência da fabricação, no Brasil, de uma LIO adequada aos cães. PEIFFER \& GAIDDON (1991) preocuparamse em propiciar uma melhor acuidade visual a cães facectomizados, adicionando-lhes uma LIO. Para isso, torna-se necessário conhecer o poder dióptrico exato do cristalino e a correlação existente entre o seu tamanho e o do animal, para que possa ser corrigida a afacia. Diante do exposto, este estudo teve por objetivo estabelecer o poder dióptrico de uma LIO, a ser implantada após facectomia, e correlacionar essa variável com o peso, o sexo e a conformação anatômica do olho, direito ou esquerdo, dos animais.

A literatura ainda é escassa nesta área da oftalmologia veterinária, mas muito se tem pesquisado sobre as técnicas cirúrgicas de facectomia e sobre a correção da afacia, sendo que o primeiro trabalho foi realizado na medicina humana, por FYODOROV et al. (1975) que calcularam o poder dióptrico de LIOs a serem aplicadas em 150 olhos e, para este fim, não consideraram a espessura corneana uma variável importante. Alguns anos mais tarde, PEIFFER \& GAIDDON (1991) relataram um estudo de 65 olhos caninos que receberam LIO, as quais variavam de 25 a 30 dioptrias (D) de poder de refração. A retinoscopia demonstrou que a maioria dos olhos estavam próximos à emetropia e que a função visual desses olhos foi superior à de pacientes afácicos. Na mesma época, DAVIDSON et al. (1991) estudaram 160 olhos caninos e observaram, por retinoscopia, que cães com LIO de 30 D necessitavam de cerca de 4,45+/-1,02D no pósoperatório. Utilizando métodos de exames diferentes, ultra-sonografia modo-A e ceratometria, em ambos os olhos de 62 cães de ambos os sexos e de várias raças, tamanhos e idades, GAIDDON et al. (1991) sugeriram que cães afácicos requerem um implante de 30 a 40D para ter emetropia. Para a realização desses exames, foi necessária a anestesia dos animais com tiamilal sódico, ou apenas sedação com cloridrato de xilazina associado, ou não, com cloridrato de quetamina.

DAVIDSON et al. (1993) relataram o uso da retinoscopia em 256 olhos de 191 cães para determinar o estado refrativo pós-facectomia e que poder de LIO implantada melhor se aproximava da emetropia em olhos pseudofácicos. A raça e o tipo constitucional dos cães não foram considerados importantes no estado refrativo final. Para a realização do exame pré-operatório, a midríase e cicloplegia foram induzidas com atropina $1 \%$ ou ciclopentolato $1 \%$ ou tropicamide $1 \%$. A retinoscopia foi realizada de zero a 99 semanas após o implante. Concluíram que o mais adequado é calcular o poder dióptrico de LIO individualmente. Caso isso não fosse possível, os autores recomendaram o uso de uma LIO de 41,5D.

GAIDDON et al. (1996) realizaram retinoscopia em dois grupos de cães, sem doenças oculares ou sistêmicas, de 35 raças diferentes. Para este exame, os olhos foram dilatados com tropicamide. O primeiro grupo contou com 74 cães normais, machos e fêmeas, com idades entre quatro meses e 13 anos, separados pelo tamanho, formato do crânio e estilo de vida. O segundo grupo foi constituído por 17 cães afácicos e pseudofácicos, com idades variando entre dois anos e meio e 11 anos. Nenhuma correlação existiu entre as medidas de retinoscopia e o formato do crânio. Consideraram que a melhor escolha seria fazer os cães emétropes individualmente ou implantar uma LIO de 41D, próxima à média de emetropia.

RUBIN \& HOPE (1996) fizeram uma revisão na oftalmologia humana sobre refração e correção. A disponibilidade do ultra-som modo-A tornou esse exame auxiliar rotineiro para determinar o poder adequado de LIOs e tem contribuído para resultados visuais excelentes após a cirurgia.

WILLIAMS et al. (1996) realizaram uma revisão da literatura oftalmológica e constataram que a cirurgia de catarata humana foi revolucionada pela introdução de LIOs para produzir um olho pseudofácico emétrope pós-cirúrgico. Apesar de muitos cães andarem bem após a facectomia, tendo apenas a percepção de vultos sem uma adequada definição de imagens, alguns pesquisadores relataram melhores resultados em testes de visão em animais com implantes de LIOs. De acordo com os autores, os cães normais geralmente são emétropes ou ligeiramente míopes. Dados obtidos na biometria, por meio de ultra-som modo-A, e na ceratometria, foram utilizados em uma fórmula específica, a qual indicou que a maioria dos cães requer um implante de aproximadamente 28D. Já a retinoscopia de olhos de cães pseudofácicos revelou que uma lente em torno de 30D parece ser necessária pela maioria dos cães. 


\section{MATERIAL E MÉTODOS}

Foram selecionados 120 cães (240 olhos), fornecidos pelo Biotério Central da UNESP Campus de Botucatu/SP, sem raça definida, sadios ao exame clínico, sendo 60 machos e 60 fêmeas, com idades variando de dois a seis anos. Realizou-se exame ocular externo, descartando-se os animais que apresentaram anormalidades do bulbo do olho.

Os animais foram separados em seis grupos segundo o peso, de acordo com os seguintes critérios: grupo 1 (20 animais pesando entre 1,0 e $5,0 \mathrm{~kg}$ ), grupo 2 (20 animais pesando entre $5,1 \mathrm{e}$ $10,0 \mathrm{~kg}$ ), grupo 3 (20 animais pesando entre $10,1 \mathrm{e}$ $15,0 \mathrm{~kg}$ ), grupo 4 (20 animais pesando entre 15,1 e $20,0 \mathrm{~kg}$ ), grupo 5 (20 animais pesando entre $20,1 \mathrm{e}$ $25,0 \mathrm{~kg}$ ) e grupo 6 (20 animais pesando acima de $25,1 \mathrm{~kg}$ ). Cada grupo, por sua vez, foi dividido em subgrupos, de 10 animais cada, de acordo com o sexo dos cães.

Realizou-se a seguinte seqüência experimental: 1) pesagem do animal, 2) medicação pré-anestésica intravenosa com Cloridrato de Levomepromazina ${ }^{1}, 1 \mathrm{mg} / \mathrm{kg}$ de peso, 3) manutenção anestésica dissociativa com $0,3 \mathrm{mg} / \mathrm{kg}$ de peso de Midazolam $^{2}$ associado a $2 \mathrm{mg} / \mathrm{kg}$ de peso de Cloridrato de Quetamina ${ }^{3}$, via intravenosa, 4) anestesia tópica com colírio de Cloridrato de Tetracaína ${ }^{4} 10 \mathrm{mg}$, 5) mensuração da curvatura corneana (ceratometria) por meio de ceratômetro $\mathrm{KM}-500^{5}$, 6) mensurações intra-oculares, utilizando a ultra-sonografia modo-A com transdutor de $10 \mathrm{Mhz}^{6}$, por meio de ecobiômetro.

As mensurações intra-oculares são comprimento axial do bulbo do olho, distância entre córnea e cápsula anterior do cristalino, comprimento axial do cristalino, distância entre cápsula posterior do cristalino e retina, cálculo do poder dióptrico da LIO necessária para correção da afacia e fornecimento de emetropia, realizado, pelo próprio aparelho, através da fórmula SRK com constante da lente de polimetilmetacrilato em 118,3. Cada uma das 10 repetições consistiu de uma média de seis mensurações.

Para o procedimento estatístico, foi utilizada a análise multivariada de perfil (MORRISON, 1990).

\section{RESULTADOS E DISCUSSÃO}

A perda do cristalino resulta em uma alta hipermetropia e comprometimento da visão. A correção pode ser feita com a utilização de lente intra-ocular (LIO), visando à restauração de visão acurada. Em um experimento desse tipo, o tamanho da amostra é importante, uma vez que servirá de base para estudos futuros, devendo ser representativa. Foram utilizados 120 cães com o intuito de diminuir o índice de erros, proporcionando uma padronização de dados estatisticamente adequada, e utilizaram-se médias de mensurações repetidas para determinar um valor o mais próximo possível do verdadeiro. Os animais não tinham raça definida, pela dificuldade em se conseguir tantos animais de uma mesma raça. Este fato é de menor importância, pois segundo GAIDDON et al. (1996), o formato do crânio das raças definidas não interfere com as medidas do bulbo do olho. Selecionaram-se animais sem doenças sistêmicas ou oftálmicas aparentes, para não haver interferência nas mensurações, como preconizado por GAIDDON $\boldsymbol{e t}$ al. (1996).

A utilização de cães machos e fêmeas teve como objetivo a verificação de possível influência deste parâmetro sobre as mensurações, como realizado por GAIDDON et al. (1991). Os animais foram separados em grupos de acordo com o peso, já que não tinham raça definida e o peso poderia influenciar nas mensurações, uma vez que aqueles mais pesados também podem ser maiores. Este tipo de separação em grupos foi preconizada por GAIDDON et al. (1991), DAVIDSON et al. (1993) e GAIDDON $\boldsymbol{e t}$ al. (1996). DAVIDSON $\boldsymbol{e t}$ al. (1993) não associaram seus achados com o tamanho dos animais examinados. GAIDDON et al. (1996) consideraram melhor avaliar os cães individualmente, devido aos diferentes tamanhos e raças que estes podem apresentar. Também foram realizadas mensurações separadas para olho direito e olho esquerdo, como sugeriram GAIDDON et al. (1991), pois esse parâmetro poderia influenciar os resultados finais.

Estabeleceu-se a idade mínima dos cães utilizados em dois anos, pois de acordo com KIRK \& BISTNER (1987), aqueles de raças pequenas têm seu crescimento cessado aos 18 meses e os de raças grandes aos 24 meses. Essa idade foi escolhida para diminuir a chance de utilizar animais com bulbos oculares ainda não totalmente formados. A idade máxima foi estabelecida em seis anos, pois KIRK \& BISTNER (1987), SLATTER (1990) e GELATT (1991) relatam que, após essa idade, começa a esclerose nuclear e aumenta significativamente o índice de doenças oftálmicas, como a catarata senil.

A medicação pré-anestésica e a anestesia dissociativa utilizadas nesse experimento foram adotadas por provocarem mínimas alterações na pressão intra-ocular, por manterem o bulbo do olho centralizado pelo tempo necessário às mensurações e por proporcionarem um retorno anestésico rápido 
nos animais, como realizado por RANZANI $\boldsymbol{e t}$ al. (1990), diferindo de GAIDDON et al. (1991). O uso de anestesia tópica, como utilizada por COTTRELL \& PETERSEN-JONES (1993), facilitou a realização do exame.

Os aparelhos utilizados para realizar as mensurações oculares foram o ceratômetro e o ecobiômetro (por meio de ultra-sonografia modo-A), como fizeram GAIDDON et al. (1991) e WILLIAMS et al. (1996). Esses aparelhos foram empregados por serem considerados importantes exames auxiliares para a determinação do poder dióptrico adequado de LIOs implantadas, conforme relataram DAVIDSON et al. (1993) e RUBIN \& HOPE (1996).

A midríase e a cicloplegia antes da realização dos exames foram preconizadas por DAVIDSON $\boldsymbol{e t} \boldsymbol{a l}$. (1993) e GAIDDON $\boldsymbol{e t} \boldsymbol{a l}$. (1996). No presente estudo, esses procedimentos não foram efetuados, pois essas condições não influenciam os resultados finais das medidas estudadas para cálculo de LIO.

Para calcular o poder dióptrico da LIO, considerou-se um erro refrativo de $-1 \mathrm{D}$, deixando os cães próximos ao seu estado fisiológico de miopia, descrito por SLATTER (1990), GUM (1991), SEVERIN (1991) e WILLIAMS et al. (1996). A espessura corneana foi desconsiderada, como preconizaram FYODOROV et al. (1975), porque suas diferenças entre os animais não é clinicamente significativa.

A comparação entre olhos direitos e esquerdos nos animais de todos os grupos experimentais revelou valores semelhantes, exceto nas fêmeas dos grupos 4 e 5 , nas quais o poder dióptrico da LIO foi significativamente maior nos olhos esquerdos, em relação aos olhos direitos (Tabela 1).

Comparando-se os olhos direitos dos machos, entre grupos, não se verificou diferença significativa entre o poder dióptrico da LIO. No entanto, para os olhos esquerdos, o poder dióptrico da LIO, para os animais dos grupos $2 \mathrm{e}$ 4 , não apresentou diferença significativa para os grupos 1,3 e 5 e foi significativamente menor que o do grupo 6. O poder dióptrico da LIO, para os animais dos grupos 1, 3, 5 e 6, não apresentou diferença significativa entre si (Tabela 1).

$\mathrm{Na}$ comparação entre grupos para os olhos direitos das fêmeas, não houve diferença significativa entre o poder dióptrico da LIO para os animais dos seis grupos; na comparação entre grupos para os olhos esquerdos das fêmeas, o poder dióptrico da LIO, para os animais dos grupos 1, 2 e 3, não apresentou diferença
Tabela 1 - Médias de 10 repetições do poder dióptrico da LIO segundo grupos de peso e sexo, para cada olho, e resultados do teste estatístico.

\begin{tabular}{|c|c|c|c|}
\hline \multirow[t]{2}{*}{ Grupo } & \multirow[t]{2}{*}{ Sexo } & \multicolumn{2}{|c|}{ Dioptrias do cristalino } \\
\hline & & Olho direito & Olho esquerdo \\
\hline Grupo 1 & Machos & ${ }_{a} 36,2^{A}$ & ${ }_{\mathrm{ab}} 36,2^{\mathrm{A}}$ \\
\hline Grupo 2 & Machos & $34,4^{\mathrm{A}}$ & $34,4^{\mathrm{A}}$ \\
\hline Grupo 3 & Machos & $34,1^{\mathrm{A}}$ & $35,7^{\mathrm{A}}$ \\
\hline Grupo 4 & Machos & $35,3^{\mathrm{A}}$ & $34,8^{\mathrm{A}}$ \\
\hline Grupo 5 & Machos & $36,3^{\mathrm{A}}$ & $37,0^{\mathrm{A}}$ \\
\hline Grupo 6 & Machos & $38,8^{\mathrm{A}}$ & b $39,8^{A}$ \\
\hline Grupo 1 & Fêmeas & $35,1^{\mathrm{A}}$ & $35,3^{\mathrm{A}}$ \\
\hline Grupo 2 & Fêmeas & $34,4^{\mathrm{A}}$ & $34,3^{\mathrm{A}}$ \\
\hline Grupo 3 & Fêmeas & $35,1^{\mathrm{A}}$ & $35,4^{\mathrm{A}}$ \\
\hline Grupo 4 & Fêmeas & $35,8^{\mathrm{A}}$ & ab $37,2^{\mathrm{B}}$ \\
\hline Grupo 5 & Fêmeas & $39,0^{\mathrm{A}}$ & $41,0^{\mathrm{B}}$ \\
\hline Grupo 6 & Fêmeas & $36,3^{\mathrm{A}}$ & ab $36,8^{\mathrm{A}}$ \\
\hline
\end{tabular}

Coeficiente de correlação de Pearson entre as variáveis olho direito e olho esquerdo: $\mathrm{r}=0,6182(\mathrm{P}<0,01)$. Para cada sexo:

Letras maiúsculas: para cada grupo, médias de olho seguidas de letras iguais não diferem significativamente $(\mathrm{P}>0,05)$;

Letras minúsculas: para cada "olho", médias de grupos seguidas de letras iguais não diferem significativamente $(\mathrm{P}>0,05)$.

significativa para os grupos 4 e 6 e foi significativamente menor que o do grupo 5 . O poder dióptrico da LIO, para os animais dos grupos 4, 5 e 6 , não apresentou diferença significativa entre si (Tabela 1).

$\mathrm{Na}$ comparação entre grupos, independente de olho, para os machos, o poder dióptrico da LIO para os animais dos grupos 2, 3 e 4 não apresentou diferença significativa para os grupos 1 e 5 e foi significativamente menor que o grupo 6; para os animais dos grupos 1, 5 e 6 não apresentou diferença significativa entre si (Tabela 2).

$\mathrm{Na}$ comparação entre grupos, independente de olho, para as fêmeas, o poder dióptrico da LIO, para os animais dos grupos 1,2 , e

Tabela 2 - Médias do poder dióptrico da LIO de acordo com os grupos de peso e sexo, e resultados do teste estatístico, independente de olho estudado.

\begin{tabular}{lcccccc}
\hline Sexo & \multicolumn{5}{c}{ Grupos } \\
\cline { 2 - 7 } & Grupo 1 & Grupo 2 & Grupo 3 & Grupo 4 & Grupo 5 & Grupo 6 \\
\hline Machos & $\mathrm{a}^{\mathrm{a} 36,2^{\mathrm{AB}}}$ & $\mathrm{a}_{\mathrm{a}} 34,4^{\mathrm{B}}$ & $\mathrm{a}^{\mathrm{a}} 34,9^{\mathrm{B}}$ & ${ }_{\mathrm{a}} 35,1^{\mathrm{B}}$ & ${ }_{\mathrm{a}} 36,7^{\mathrm{AB}}$ & ${ }_{\mathrm{a}} 39,3^{\mathrm{A}}$ \\
Fêmeas & $\mathrm{a} 35,2^{\mathrm{B}}$ & $\mathrm{a}^{\mathrm{a}} 34,4^{\mathrm{B}}$ & $\mathrm{a}_{\mathrm{a}} 35,3^{\mathrm{B}}$ & ${ }_{\mathrm{a}} 36,5^{\mathrm{AB}}$ & ${ }_{\mathrm{b}} 40,0^{\mathrm{A}}$ & $\mathrm{b}^{3} 36,6^{\mathrm{AB}}$ \\
\hline
\end{tabular}

Letras maiúsculas: para cada sexo, médias de grupos seguidas de letras iguais não diferem significativamente $(P>0,05)$;

Letras minúsculas: para cada grupo, médias de sexo seguidas de letras iguais não diferem significativamente $(\mathrm{P}>0,05)$. 
3, não apresentou diferença significativa para os grupos 4 e 6 e foi significativamente menor que o grupo 5; para os animais dos grupos 4, 5 e 6, não apresentou diferença significativa entre si. $\mathrm{Na}$ comparação entre machos e fêmeas, o poder dióptrico da LIO apresentou diferenças apenas nos animais do grupo 5, no qual foi significativamente menor nos olhos dos machos, em relação às fêmeas, e nos animais do grupo 6, no qual foi significativamente maior nos olhos dos machos, em relação às fêmeas (Tabela 2).

A variação apresentada no poder dióptrico das LIOs para machos e fêmeas é superior (Tabela 1) aos valores superiores aos sugeridos por PEIFFER \& GAIDDON (1991), 25,0 a 30,0D, SAMUELSON (1991), ao redor de 30,0D, WILLIAMS et al. (1996), 30,0D, e semelhantes ao recomendado por CARNEIRO FILHO (1997), 42,0D. Os resultados obtidos foram ligeiramente superiores àqueles de DAVIDSON et al. (1991), segundo os quais, cães com LIO de 30D necessitavam de 4,45+/-1,02D no pós-operatório para alcançar a emetropia, e próximos aos de GAIDDON et al. (1991), que sugeriram um implante de 30 a 40D. Já DAVIDSON et al. (1993) e GAIDDON $\boldsymbol{e t}$ al. (1996) aconselharam, caso não fosse possível realizar a ceratometria e biometria de rotina, o implante de uma LIO de 41,5 ou 41D, respectivamente, que são valores compatíveis com os encontrados neste experimento. Porém, torna-se difícil considerar qualquer um desses autores como referência, uma vez que, em seus experimentos, não foi realizada uma padronização adequada, seja em relação aos animais utilizados ou às variáveis mensuradas.

De acordo com os resultados obtidos, há uma semelhança entre a variável mensurada quando comparada entre os sexos e entre os olhos, direito e esquerdo.

\section{CONCLUSÃO}

Nas condições experimentais adotadas concluiu-se que: o poder dióptrico de uma LIO mostra valores semelhantes entre olho direito e olho esquerdo, dentro de um mesmo sexo, exceto para fêmeas de 15,1 a $25 \mathrm{~kg}$ de peso, nas quais os olhos esquerdos apresentam valores maiores; os valores encontrados para o poder dióptrico de uma LIO para machos e fêmeas, dentro de um mesmo grupo de peso, são semelhantes, exceto para animais acima de $20,1 \mathrm{~kg}$; os valores encontrados para o poder dióptrico de uma LIO são diferentes quando comparados entre os animais dentro do mesmo sexo, independente de olho. Entre machos, animais de 5,1 a $20 \mathrm{~kg}$ apresentam-se com poder dióptrico diferente quando comparados com aqueles acima de $25,1 \mathrm{~kg}$ de peso. Fêmeas de 1 a $15 \mathrm{~kg}$ apresentam-se diferentes daquelas entre $20,1 \mathrm{a} 25 \mathrm{~kg}$ de peso.

$O$ principal parâmetro que influencia o provável poder dióptrico de uma LIO no cão é o peso.

\section{FONTES DE AQUISIÇÃO}

${ }^{1}$ Neozine - Rhodia - Grupo Rhône-Poulenc - São Paulo/SP.

${ }^{2}$ Dormonid - Produtos Roche Químicos e Farmacêuticos S.A. Rio de Janeiro/RJ.

${ }^{3}$ Ketalar - Parke-Davis - Aché Laboratórios Farmacêuticos S.A. - Guarulhos/SP.

4 Anestésico "Oculum" colírio - Labs. Frumtost S.A. Guarulhos/SP

${ }^{5}$ Ceratômetro NIDEK.

${ }^{6}$ Echoscan US-800, NIDEK.

\section{REFERÊNCIAS BIBLIOGRÁFICAS}

CARNEIRO FILHO, L. Manual de oftalmologia veterinária: um guia prático para clínicos veterinários. São Paulo : Roca, 1997. Exame oftálmico: p.3-19.

COTTRELL, B.D., PETERSEN-JONES, S.M. Special examination techniques. In: PETERSEN-JONES, S.M., CRISPIN, S.M. Manual of small animal ophthalmology. Shurdington : British Small Animal Veterinary Association, 1993. Chap.2, p.27-43.

DAVIDSON, M.G., NASISSE, M.P., JAMIESON, V.E., $\boldsymbol{e t}$ al. Phacoemulsification and intraocular lens implantation: a study of surgical results in 182 dogs. Prog Vet Comp Ophthalmol, v.1, p.233-238, 1991.

DAVIDSON, M.G., MURPHY, C.J., NASISSE, M.P., et al. Refractive state of aphakic and pseudophakic eyes of dogs. Am J Vet Res, v.54, n.1, p.174-177, 1993.

FYODOROV, S.N., GALIN, M.A., LINKSZ, A. Calculation of the optical power of intraocular lenses. Invest Ophthalmol, v.14, n.8, p.625-628, 1975.

GAIDDON, J., ROSOLEN, S.G., STERU, L., et al. Use of biometry and keratometry for determining optimal power for intraocular lens implants in dogs. Am J Vet Res, v.52, n.5, p.781-783, 1991.

GAIDDON, J., BOUHANA, N., LALLEMENT, P.E. Refraction by retinoscopy of normal, aphakic, and pseudophakic canine eyes: advantage of a 41-diopter intraocular lens? Vet and Comp Ophthalmol, v.6, n.2, p.121 - 124, 1996.

GELATT, K.N. Veterinary ophthalmology. 2. ed. Philadelphia : Lea \& Febiger, 1991. The canine lens: p.429460.

GUM, G.G. Physiology of the eye. In: GELATT, K.N. Veterinary ophthalmology. 2. ed. Philadelphia : Lea \& Febiger, 1991. Chap.2. p.124-161.

KIRK, R.W., BISTNER, S.I. Manual de procedimentos e tratamento de emergência em medicina veterinária. 3. ed. São Paulo : Manole, 1987. p.868. 
MORRISON, D.H. Multivariate statistical methods. 3. ed New York : Mc Graw-Hill, 1990. 495p.

PEIFFER, R.L., GAIDDON, J. Posterior chamber intraocular lens implantation in the dog: results of 65 implants in 61 patients. J Am Anim Hosp Assoc, v.27, p.453-462, 1991.

RANZANI, J.J.T., CASTRO, G.B., AGUIAR, A.J.A., $\boldsymbol{e}$ t $\boldsymbol{a}$. Emprego do midazolam na avaliação da PIO em cães. In: CONGRESSO BRASILEIRO DA ASSOCIAÇÃO NACIONAL DE CLÍNICOS VETERINÁRIOS DE PEQUENOS ANIMAIS, 13, 1990, Gramado. Anais... Gramado: ANCLIVEPA-RS, 1990. p.30.

RUBIN, M.L., HOPE, G.M. Optics and refraction: a review. Ophthalmology, v.103, n.8, p.102-108, 1996.
SAMUELSON, D.A. Ophthalmic embryology and anatomy. In: GELATT, K.N. Veterinary ophthalmology. 2.ed. Philadelphia : Lea \& Febiger, 1991. Chap.1. p.3-123.

SEVERIN, G.A. Manual de oftalmologia veterinaria. Buenos Aires : Hemisferio Sur, 1991. Exploracion del ojo: p.1-19.

SLATTER, D. Fundamentals of veterinary ophthalmology. 2.ed. Philadelphia : Saunders, 1990. Structure and function of the eye: p. $1-17$.

WILLIAMS, D.L., BOYDELL, I.P., LONG, R.D. Current concepts in the management of canine cataract: a survey of techniques used by surgeons in Britain, Europe and the USA and a review of recent literature. Vet Rec, v.13, p.347-353, 1996. 